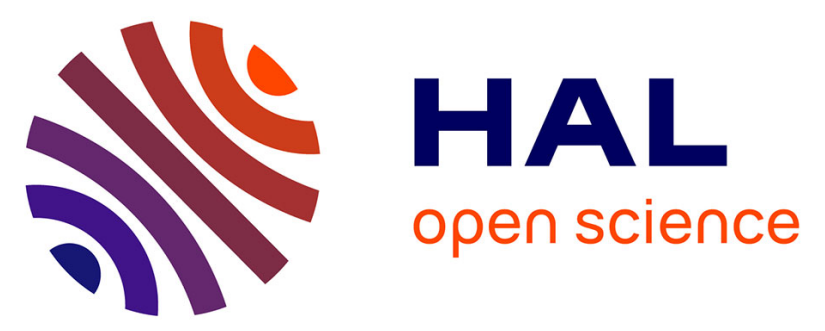

\title{
The anatomo-functional organization of the hyperdirect cortical pathway to the subthalamic area using in vivo structural connectivity imaging in humans
}

Gizem Temiz, Sophie B. Sébille, Chantal François, Eric Bardinet, Carine Karachi

\section{To cite this version:}

Gizem Temiz, Sophie B. Sébille, Chantal François, Eric Bardinet, Carine Karachi. The anatomofunctional organization of the hyperdirect cortical pathway to the subthalamic area using in vivo structural connectivity imaging in humans. Brain Structure and Function, 2019, 1, Epub ahead of print. 10.1007/s00429-019-02012-6 . inserm-02426587

\section{HAL Id: inserm-02426587 https://www.hal.inserm.fr/inserm-02426587}

Submitted on 2 Jan 2020

HAL is a multi-disciplinary open access archive for the deposit and dissemination of scientific research documents, whether they are published or not. The documents may come from teaching and research institutions in France or abroad, or from public or private research centers.
L'archive ouverte pluridisciplinaire HAL, est destinée au dépôt et à la diffusion de documents scientifiques de niveau recherche, publiés ou non, émanant des établissements d'enseignement et de recherche français ou étrangers, des laboratoires publics ou privés. 
The anatomo-functional organization of the hyperdirect cortical pathway to the subthalamic area using in vivo structural connectivity imaging in humans

Gizem Temiz ${ }^{1,2}$, Sophie B. Sébille ${ }^{1,2}$, Chantal Francois ${ }^{1}$, Eric Bardinet ${ }^{1,2}$, Carine Karachi ${ }^{1,3}$

${ }^{1}$ Sorbonne Universités, UPMC Univ Paris 06, CNRS, INSERM, APHP GH Pitié-Salpêtrière, Institut du cerveau et de la moelle épinière (ICM), F-75013 Paris, France

${ }^{2}$ Center for Neuroimaging Research - CENIR, Paris, France

${ }^{3}$ AP-HP, Hôpital de la Pitié-Salpêtrière, Department of Neurosurgery, Paris, France

Correspondance to: Chantal Francois, Sorbonne Universités, UPMC Univ Paris 06, CNRS, INSERM, APHP GH Pitié-Salpêtrière, Institut du cerveau et de la moelle épinière (ICM), F-75013 Paris, France

$\underline{\text { ch.francois.deligne@gmail.com }}$

\section{Acknowledgements}

This work was supported by 'Investissements d'avenir' (Investing in the future programme ANR-10-IAIHU-O6). G. Temiz was supported by the "Fondation pour la Recherche" Médicale (FRM) (Project:DIC20161236441) and by Boston Scientific. The authors would like to thank Max Westby for language editing.

\section{Declarations of interest:}

None 


\begin{abstract}
The subthalamic nucleus (STN) receives direct cortical inputs which constitute the so-called hyperdirect pathway. In monkeys, motor cortices innervate the whole extent of the STN whereas limbic cortices innervate only its anteromedial part extending more medially outside the nucleus. Tractography studies in humans have also identified motor cortical inputs to the STN, but little is known about the associative and limbic cortical projections. Therefore, the aim of this study was to investigate the anatomo-functional organization of the cortical projections to the STN and to the adjacent medial subthamic region (MSR). We used diffusion weighted imaging-based tractography acquired from 30 subjects from the Human Connectome Project. We performed a whole-brain probabilistic tractography using MrTrix and extracted streamlines of interest between 39 cortical masks and both the STN and the MSR to provide track-density maps. Agglomerative clustering method was used to classify the voxels of the regions of interest. We found that the STN receives major inputs from the sensorimotor cortices and few inputs from the limbic cortices. On the other hand, the MSR receives mainly cortical limbic projections and few from the sensorimotor cortices. Weak connections were found between the associative cortices and both the STN and the MSR. We found a dominant motor cluster located in the posterolateral STN, a limbic cluster located medially in the MSR, and an intermediate motor-limbic cluster in between. Our findings show that the hyperdirect pathway is anatomo-functionally organized with a poor participation of associative cortices.
\end{abstract}

Key words: cortical projections, subthalamic area, diffusion weighted imaging-based tractography, sensorimotor cortex, limbic cortex, human

List of abbreviations:

BA, Brodmann areas

DBS, deep brain stimulation

DWI, diffusion weighted imaging

FA, fractional anisotropy 
FOD, fiber orientation distributions

MSR, medial subthalamic region

$\mathrm{MR}$, magnetic resonance

MRI, magnetic resonance imaging

PD, Parkinson's disease

ROI, region of interest

STN, subthalamic nucleus

LH, Left hemisphere

$\mathrm{RH}$, right hemisphere. 


\section{Introduction}

Deep brain stimulation (DBS) of the subthalamic nucleus (STN) alleviates remarkably motor symptoms of patients with Parkinson's disease (PD) that respond to dopaminergic medication (Limousin et al. 1998). In PD patients, it was also shown that STN DBS can induce psychiatric symptoms such as hypomanic status especially when the chosen contacts of the DBS electrodes are located in the anteromedial part of the nucleus (Kim et al. 2012; Welter et al. 2014). Moreover, anteromedial STN DBS is also used with success to alleviate symptoms of patients with severe and resistant obsessive-compulsive disorders (Mallet et al. 2008). These differential effects obtained along the anteroposterior axis of the STN were specifically attributed to its anatomo-functional subdivision into three territories: a sensorimotor territory located in the posterolateral STN that encodes motor and sensitive information, an associative territory located in the central STN that encodes cognitive information and a limbic territory in the anteromedial STN that encodes emotional and motivational information (Haber et al. 1993; Shink et al. 1996; Francois et al. 2004; Karachi et al. 2005).

This tripartite subdivision was supported by numerous experimental data obtained in several animal species showing that three anatomo-functional territories of the cortico-striato-pallidal pathway are preserved in the pallido-subthalamic projections (Haber et al. 2000; Parent and Hazrati 1995; Shink et al. 1996). It was also shown that these anatomo-functional territories, especially the associative and the limbic, have no clear-cut boundaries, but are separated by functional gradients, as observed using tract tracing in monkey (Karachi et al. 2005). This specific partition of the STN was also confirmed functionally in awake monkey. Microinjections of the GABA antagonist (bicuculline) into the posterolateral STN induces abnormal movements such as hemiballismus whereas microinjections into the anteromedial STN induces stereotyped behaviors (Crossman et al. 1984; Karachi et al. 2009). Based on this segregation given by external pallidal afferences, it is thus possible to define a sensorimotor part located in the posterolateral STN, the associative part in the central STN, and the limbic part in the anteromedial tip.

The STN also receives direct excitatory inputs from the cortex, referred as the hyperdirect pathway (Nambu et al. 1996). The hyperdirect pathway characterizes a monosynaptic connection between the different cortical areas, whatever their function, and the STN. In rat, the primary motor cortex, the granular insular cortex, the prelimbic-medial orbital areas of the prefrontal cortex, and the anterior cingulate cortex are the major sources of cortical inputs to the STN with no projection originating from the post-central cortex (Afsharpour 1985; Canteras et al. 1990; Maurice et al. 1998). However, anatomo-functional comparisons between cortical areas of 
rodent and primate are difficult because of the relative absence of associative cortices in rodent. In monkeys, numerous tract-tracing studies have shown that all motor cortices (Brodmann areas (BA) 4, 6, Supplementary Motor Area (SMA), pre-SMA, and motor cingulate cortex (BA 24c)) are at the origin of the hyperdirect pathway (Künzle 1975; 1978; Hartman-von Monakow et al. 1978; Jurgens 1984; Nambu et al. 1996; 1997; Takada et al. 2001; Haynes and Haber 2013). All these studies suggest that within the STN, the motor cortical area occupies the whole extent of the STN (Nambu et al. 1996; 1997), the ventral part of the nucleus being devoted to the frontal eye field and the supplementary eye field projections (Künzle and Akert 1977; Hartman-von Monakow et al. 1978; Jurgens 1984; Shook et al. 1991).

In comparison, few studies indicate if the limbic and above all the associative cortices could compose the hyperdirect pathway. Thus in monkey, only the limbic cortical BA 14 and the associative cortical BA 46 and 9 were reported to project to the STN (Künzle 1978; Hartman-von Monakow et al. 1978; Haynes and Haber 2013). These limbic cortical inputs innervate not only the anteromedial part of the STN, but extend to an adjacent region medially located (Haynes and Haber 2013). Indeed, the anteromedial limit of the STN with the adjacent structures is difficult to delineate especially in rat, leading the tip of the STN open (Hammond and Yelnik 1983). This medial area, also named parasubthalamic nucleus (Goto and Swanson 2004), continues medially towards the medial line where the so-called "triangle de Sano" was defined (Sano et al. 1966) and sometimes referred as the posterior hypothalamus (Sano 1962). This medial subthalamic region contains many tracts such as the Forel's fields (Forel 1877), the pallido-thalamic, nigro-thalamic or mammillo-tegmental tracts (Neudorfer and Maarouf 2018). It is interesting to note that the Sano triangle is an old surgical target for treating violent behavior seen in many psychiatric disorders with interesting clinical results using lesioning and more recently DBS (Sano et al 1966; 1970; Franzini et al. 2013). Aggressive behavior was also observed in a parkinsonian patient treated with STN DBS when the stimulating contact of the electrode was located in this medial subthalamic area (Bejjani et al. 2002). Based on these clinical observations, it looks probable that this medial subthalamic region (MSR) could receive important limbic information notably from the cortex.

Data obtained in human using diffusion weighted imaging-based (DWI) tractography have also suggested that the motor cortex, but also the prefrontal and limbic orbitofrontal cortices projected to the STN (Aravamuthan et al. 2007; Brunenberg et al. 2012; Lambert et al. 2012; Accola et al. 2014; Mulder et al. 2014; Avecillas-Chasin et al. 2016; Petersen et al. 2016, Akram et al. 2017b). The evidence of a tripartite subdivision of the STN has been the subject of debates and challenged (Alkemade and Forstmann 2014; Lambert et al. 
2015), and it is still unclear whether the whole associative cortical areas are connected to the human STN, and to what extent the different anatomo-functional cortical projections are segregated within the nucleus.

Here, we aim to determine which cortical areas composed the hyperdirect pathway and search for an anatomo-functional subdivision of the STN based on cortical functions in human. For that, we analyzed the connectivity of the whole cortex classified into three groups based on functions (sensorimotor, associative and limbic) with both the STN and the MSR in 30 healthy subjects. We used DWI in order to provide new neuroimaging evidences of the anatomo-functional organization of the hyperdirect pathway to the STN area in human.

\section{Material and methods}

\section{Volunteers}

Data from 30 healthy volunteers (12 male, average age 30 years) from the Human Connectome Project (HCP) were included in this study (Van Essen et al. 2013). Selected subjects were those already chosen for a previous study (Sébille et al. 2017). HCP experiments were performed in accordance with relevant guidelines and regulations and the experimental protocol was approved by the Institutional Review Board. Our choice to use 30 healthy volunteers is based on three criteria. First, standard deviations for the inter-volunteer streamline numbers being small, the streamline numbers were considered as a homogeneous population. Second, volunteers used from the HCP database are young adults with small ventricles and no cortical atrophy, which provides an excellent alignment of our deformable YeB atlas onto each MRI volunteer's data. These features also enable us to reliably segment the different cortical and subcortical areas. Third, a population size of $30 \mathrm{HCP}$ healthy volunteers has already been used in other tractography studies (see for example Wang et al. 2019, Ugurlu et al. 2018).

\section{Data processing}

The methodological pipeline used to estimate DWI-based connectivity of the whole cortex to the STN and the MSR is summarized in Supplementary Fig. 1. HCP data acquisition specifications and preprocessing steps that we used were explained in previous studies (Glasser et al. 2013; Sotiropoulos et al. 2013; Andersson and Sotiropoulos 2015) and detailed in Supplementary Methods (Image acquisition and preprocessing). 
The STN was segmented using our in-house 3D histological and deformable atlas (YeB atlas) (Bardinet et al. 2009), the MSR were manually segmented by two anatomical experts (CK, CF), and cortical areas were segmented using the Brodmann atlas projected to the native space of our subjects (See Supplementary Methods, segmentation of regions of interest). Diffusion data were processed with the MRtrix (http://www.mrtrix.org) software and we estimated the distribution of fiber orientations (FOD) present within each voxel using the multi-shell multi-tissue Constrained Spherical Deconvolution (MSMT-CSD) framework (Dhollander et al. 2016; Jeurissen et al. 2014). We used a whole brain probabilistic tractography in a multi-shell multi-tissue framework because of its greater sensitivity for the detection of crossing and curving fibers (Tournier et al. 2012; Wilkins et al. 2015; Petersen et al. 2016). Whole brain probabilistic tractography was run in the subject's native space with the following parameters: 100 million streamlines, step length of $0.1 \mathrm{~mm}$, curvature threshold of $45^{\circ}$, minimum tract length of $6 \mathrm{~mm}$, maximum tract length of $250 \mathrm{~mm}$ and fiber orientation distribution amplitude cut-off of 0.1. Spherical-deconvolution Informed Filtering of Tractograms (SIFT) was applied to reduce the number of streamlines to 10 million to provide a biologically meaningful estimate of structural connection density by removing false positive tracts (Tournier et al. 2010; Smith et al. 2013) and reducing tractography biases (Maier-Hein et al. 2017).

78 tracts were extracted between 39 cortical masks and both the STN and the MSR for each subject, for each hemisphere. In order to avoid streamlines that traverse basal ganglia before reaching the STN (corresponding to the direct and indirect pathways), and thus to isolate hyperdirect cortico-STN fibers, exclusion masks were applied to the striatum, as well as the internal and external pallidum. The cerebral peduncles (CP) were used as exclusion masks in order to avoid the pyramidal tract. Cross exclusion between STN and MSR was also applied during the extraction of streamlines of interests. These exclusion masks were computed using an automatic atlas-based method, and we have checked that an alternative careful manual delineation of these masks on three random subjects did not produce statistically significant changes.

Track-density maps were calculated for all reconstructed pathways in subject native anatomical space (Calamante et al 2010). Sub-parcellation of the STN and the MSR was then generated by hierarchical agglomerative clustering using Ward's method (Gan et al. 2007) as previously used successfully by Lambert et al. (2012) (See Supplementary Methods, Hierarchical Agglomerative Classification).

\section{Statistical analysis}


In order to quantify the connectivity differences of the STN and MSR, we calculated the mean number of streamlines, expressed as a percentage, between the cortical areas and both the STN, and the MSR for each subject and averaged across 30 subjects. Comparison of sensorimotor, associative, limbic projections were also performed with the sum of the mean streamline numbers for the whole of the sensorimotor, associative and limbic cortices separately. In these cases, the sum of standard deviations was calculated as the square root of the sum of the variances. Comparison of cortex-STN connectivity and cortex-MSR connectivity results were performed using the Wilcoxon signed-rank test (MATLAB 2016b), variability being expressed as standard deviation.

\section{Results}

\section{Cortical connectivity to the STN}

All the cortical projections to the STN are listed in Table 1 and the main cortical projections can be visualized in Fig. 1.

Sensorimotor cortical areas were highly connected to the ipsilateral STN. The most connected cortical areas for both hemispheres were the premotor cortex BA 6 (39.98 \% for right hemisphere (RH), $36.65 \%$ for left hemisphere (LH)) and the primary motor cortex BA4 (28.61\% for $\mathrm{RH}$, and $31.56 \%$ for $\mathrm{LH})$ compared to all other sensorimotor, associative and limbic cortices across the 30 subjects. A weaker sensorimotor connection was observed with the frontal eye field BA $8(0.58 \%$ for $\mathrm{RH}$, and $0.89 \%$ for $\mathrm{LH})$ and with the primary somatosensory cortex BA 1-2-3 (10.77\% for RH, $13.73 \%$ for $\mathrm{LH})$ compared to the other sensorimotor areas.

Weak connectivity was obtained with the associative cortices, even for the most connected ones such as the parietal somatosensory cortices BA 5 (3.51\% for RH, $2.18 \%$ for $\mathrm{LH})$ and BA 7 (1.43 \% for RH, $0.67 \%$ for LH). The other associative cortices had very weak connectivity, characterized by streamline numbers inferior to $0.5 \%$. They were in descending order : BA 43, 9, 40, 39, 19, 37, 44, 46, 20, 21, 22, 10, 47, 29, 45, 18, 42, 38, 41, 17 for the RH, and BA 40, 9, 43, 19, 39, 44, 37, 46, 20, 45, 22, 18, 10, 29, 42, 47, 41, 21, 38, 17 for the LH.

The limbic projections to the STN mainly concerned the agranular retrolimbic cortex BA 30 (10.46\% for RH, $5.85 \%$ for LH), the perirhinal cortex BA 35 (3.55\% for RH, $1.63 \%$ for LH) and the insular cortex BA 48 ( $0.81 \%$ for RH, $1.02 \%$ for LH). The other limbic cortices were weakly connected to the STN with streamline numbers inferior to $0.5 \%$. They were in descending order : BA 34, 32, 23, 25, 27, 36, 26, 28, 11, 24 for the RH, and BA 27, 32, 23, 34, 26, 25, 24, 36, 11, 28 for the $\mathrm{LH}$. 


\section{Cortical connectivity to the MSR}

All the main cortical projections to the MSR are listed in Table 1 and the main projections can be visualized in Fig. 2.

The sensorimotor cortices were weakly connected to the MSR. The highest connectivity was obtained with the premotor cortex BA 6 (4.88\% for RH, $7.43 \%$ for $\mathrm{LH})$ and with the primary motor cortex BA $4(1.17 \%$ for RH, $3.24 \%$ for $\mathrm{LH}$ ) compared to other sensorimotor areas such as the frontal eye field oculomotor area, BA $8(0.72 \%$ for RH, $0.93 \%$ for $\mathrm{LH})$ or the somatosensory cortex BA $1-2-3(0.36 \%$ for RH, $0.46 \%$ for LH).

The associative cortices had the lowest connectivity with the MSR compared to sensorimotor and limbic cortices, with the inferior temporal cortex BA 20 (3.78\% for RH, $3.24 \%$ for LH), and the posterior inferior temporal gyrus BA 37 (1.61\% for RH and $1.58 \%$ for $\mathrm{LH})$ being the most connected associative areas. Moreover, the other weak connected associative cortices, with streamline numbers below $0.5 \%$, were in descending order: BA 9, 46, 19, 5, 7, 18, 43, 17, 21, 38, 39, 10, 29, 39, 40, 45, 22, 47, 41, 42 for the RH, and BA $9,5,7,46,44,40,19,18,45,38,43,17,39,47,10,21,22,42,29,41$ for the LH.

The most connected limbic cortical area with the MSR was the subgenual cingulate cortex BA 25 ( $49.86 \%$ for the RH, $44.56 \%$ for the $\mathrm{LH}$ ) compared to the other limbic cortical areas across the 30 subjects. The other limbic cortical projections consisted of streamlines between the MSR and the entorhinal cortex BA 34 (13.52\% for the RH, $23.43 \%$ for the $\mathrm{LH})$, the perirhinal cortex BA 35 (7.69\% for the RH, $5.56 \%$ for the $\mathrm{LH})$, the agranular retrolimbic area BA 30 (5.40\% for the RH, $2.68 \%$ for the LH), the parahippocampal area BA 27 ( $4.98 \%$ for the RH, $1.45 \%$ for the $\mathrm{LH})$, and the insular cortex BA 48 (3.40\% for the RH, $2.57 \%$ for the $\mathrm{LH}$ ). The mean connectivity of other limbic cortices was much weaker (less than $1 \%$ streamlines). In descending order, they consisted of BA 36, 32, 28, 23, 24, 26, 11 for both hemispheres.

\section{Comparison of cortical connections to the STN and the MSR}

The percentage of average number of streamlines connecting all the sensorimotor cortices to the STN (79.49 \% for the RH, $82.39 \%$ for the $\mathrm{LH})$ was much higher $(\mathrm{p} \leq 0.001)$ than those connecting the MSR $(7.71 \%$ for the RH, 14.91\% for the LH) (Fig. 3). Significant results were also observed for each of the BA 1-2-3, 4 and 6 for both hemispheres ( $\mathrm{p} \leq$ 0.001) (Fig. 4 A-B, Fig. 5), with a density gradient for the sensorimotor projections from the STN to the MSR. 
We observed only very few numbers of streamlines connecting the associative cortical areas as a whole to both the STN (4.78 \% for the RH, $7.72 \%$ for the LH) and the MSR (10.28\%, for the RH, $8.97 \%$ for the LH) (Fig. 3). We observed a higher density of associative connections with the STN than with the MSR for BA 5, 7, $37,39,40,20$ for both hemispheres, and the opposite for the right BA 22 ( $\mathrm{p} \leq 0.001)$. Weaker connectivity values were also obtained for the left BA $38(\mathrm{p} \leq 0.01)$ and left BA $29(\mathrm{p} \leq 0.05)$, which were more connected to the MSR, whereas BA 19 ( $\mathrm{p} \leq 0.01)$, left BA 22 and 29, and right BA 44 and $19(\mathrm{p} \leq 0.05)$ were more connected to the STN (Fig. 4 C-D, Fig. 5; Table 1).

We found that the MSR showed a much higher connectivity with the limbic cortices (76.03 \% for LH; $81.88 \%$ for RH) than the STN itself $(9.5 \%$ for the $\mathrm{LH}, 15.20 \%$ for the RH) $(\mathrm{p} \leq 0.001)($ Fig. 3$)$. The average numbers of streamlines connecting the MSR were significantly higher with BA 25, 28, 34, 27,36 for both hemispheres, with the left BA $30(p \leq 0.001)$, the left BA $32(p \leq 0.01)$, and the right BA 30 and $32(p \leq 0.05)$

(Fig. 4 E-F, Fig. 5; Table 1). These limbic projections were organized in an ascending gradient from the STN to the med STN.

\section{Clustering of the cortical connectivity}

We identified 3 clusters with distinct patterns of connectivity between the cortex and the STN and the MSR. The connectivity profiles and the spatial distribution of these 3 clusters were similar across the 30 subjects (Fig. 6 A-B).

Voxels involved in the first cluster had a highly connected profile with sensorimotor cortices BA 1-2-3, BA 4 and BA 6, and these voxels were located in the posterolateral part of the STN for all subjects. However, the limit of this cluster within the nucleus was variable among the subjects. A gradient of sensorimotor cortical projections existed, the posterolateral STN being the most innervated and the MSR being the least. Connectivity profiles of voxels involved in the second cluster showed connectivity with the limbic (BA 30, 34) and motor cortices (BA 6, 4), and these voxels were located in the anteromedial part of the STN and in the MSR (Fig. 6 A-B). Voxels involved in the third cluster were mainly connected to the limbic cortices BA 25 and BA $30,34,35$ and were located in the medial part of the MSR. Here, a gradient of cortical limbic projections was found between the anteromedial STN and the MSR, with the highest connectivity being with the medial part of the MSR. 
In conclusion, based on density maps of cortical projections and clustering, two anatomo-functional territories could be identified within the STN: a sensorimotor territory in the posterolateral part and a limbic territory at the anteromedial tip of the nucleus with no sharp boundaries in between.

\section{Discussion}

Our findings show that sensorimotor cortical projections to the human STN are concentrated in the posterolateral, while limbic projections terminate at the anteromedial tip, with no sharp boundaries in between. In contrast, the MSR is mainly innervated by limbic cortical projections, especially medially, and more weakly by sensorimotor projections. Therefore, sensorimotor cortical projections are positively graded from the MSR to the posterolateral STN whereas limbic cortical projections are positively graded from the posterolateral STN to the medial part of the MSR. Only weak connectivity was found between all associative cortices and both the STN and the MSR.

\section{The anatomo-functional territories of the subthalamic area}

Our results show that the number of streamlines connecting the sensorimotor cortex to the STN was the strongest component of the hyperdirect pathway compared to associative and limbic cortices, with a greater number of streamlines originating from the premotor cortex BA 6 than those originating from the primary motor cortex BA 4. This finding is in accordance with our current anatomical knowledge of the motor cortico-subthalamic projection in rodents (Afsharpour 1985; Canteras et al. 1990) and monkeys (see for review Parent and Hazrati 1995). Moreover, we found a dominant motor cluster for the 30 subjects located in the posterolateral STN that correspond to STN voxels highly connected with sensorimotor cortices. However, the boundary of this cluster that ends more or less towards the center of the nucleus is variable between individuals. This posterolateral position of the STN sensorimotor anatomo-functional territory based on cortical afferences matches with the delineation of the sensorimotor territory defined by external pallidal inputs previously described in monkey (Shink et al. 1996; Francois et al. 2004; Karachi et al. 2005). However, it is important to note that we also found some projections of sensorimotor cortices that occupied the whole anteroposterior extent of the STN even if the projections are much weaker anteriorly. Thus, sensorimotor cortical projections provide a descending gradient from posterolateral to anteromedial STN. Indeed, we identified a second cluster among the 30 subjects that connected to both motor and limbic cortices that occupied an anteromedial location within the 
nucleus and extended to the MSR. This result is in line with the findings obtained in the monkey showing that even if the primary motor cortex BA 4 terminates in the posterolateral STN, the supplementary motor area and the premotor cortex BA 6 send overlapping inputs to the anteromedial STN (Nambu 1996, 1997; Coudé et al. 2018).

Our results also show that the STN receives cortical projections from the dorsolateral prefrontal associative cortices BA 9 and 46, as already reported in monkey (Haynes and Haber 2013) and also from other areas such as parietal somatosensory cortices BA 5 and 7. It is true that the connectivity of these two associative areas (BA 5 and 7) to the STN area could be overestimated since they are adjacent to the sensorimotor area BA 4 that is highly connected to the STN.

Our results strongly suggest that the centrally located associative anatomo-functional territory of the STN is only defined by its external pallidal inputs, as demonstrated by tracing studies in monkeys (Haber et al. 1993; Shink et al. 1996; Francois et al. 2004. Karachi et al. 2005) and that associative cortico-subthalamic inputs do not significantly participate in the cortico-subthalamic hyperdirect pathway in primates.

In contrast, our data confirm the existence of direct limbic cortical inputs to the STN, occupying its anteromedial part, as previously described in rodents (Maurice et al. 1998) and monkeys (Haynes and Haber, 2013). In particular, we showed that the agranular retrolimbic cortex BA 30 and the perirhinal cortex BA 35 project to the STN as already reported in monkeys (Künzle 1978; Hartman-von Monakow et al. 1978; Haynes and Haber 2013), and in humans using DWI (Avecillas-Chasin et al. 2016), whereas the other limbic cortical areas were only weakly connected. However, in terms of projection densities, the major limbic cortical inputs do not project to the anteromedial STN but to the MSR and more precisely to its medial part with a gradient of projections between the STN to the MSR, with the subgenual cingulate cortex (BA 25), parahippocampal cortex (BA 27), entorhinal cortex (BA 34), perirhinal cortex (BA 35), and insular cortex (BA 48) not preferentially innervating the STN but the MSR. This finding confirms that the MSR is highly connected to the limbic system as already shown in monkeys (Veazey et al. 1982). This new result in the human matches with a previous hypothesis based on tracing studies on monkeys in which a re-definition of the boundaries of the STN with the inclusion of, at least a part of the MSR has been proposed based on the topography of terminals originating from the limbic cortices (Haynes and Haber 2013). Moreover, it is well known that subthalamic dendrites cross the mediodorsal border of the nucleus (Hammond and Yelnik 1983), leading to the possibility of receiving afferent fibers devoted to adjacent neurons. It was also recently shown in monkey that many cortical axons cross the STN 
and terminate in the MSRmedial STN area using single-axon tracing (Coudé et al. 2018). Taken together, these findings highlight the fact that the anteromedial STN boundaries are difficult to determine in term of cortical limbic projections.

Our DWI data provide new evidence that the classical tripartite subdivision of the human STN defined by external pallidal inputs does not match with the delineation of anatomo-functional territories based on cortical afferences. Indeed, only two anatomo-functional cortico-subthalamic territories could be delineated based on the hyperdirect pathway: a sensorimotor territory in the posterolateral STN and a limbic territory in the anteromedial STN with no sharp boundary between them.

\section{Methodological considerations:}

Previous DWI studies in humans which reported the distribution of numerous prefrontal inputs to the STN with a convergence and areas of overlap (Aron et al. 2007; Lambert et al. 2012, Accolla et al. 2014; Avecillas-Chasin et al. 2016, Akram et al. 2017b). This discrepancy with our findings could be explained by several methodological considerations. First, we excluded cortical streamlines traversing the other basal ganglia nuclei in order to avoid connectivity of the direct and indirect pathways to the STN. We followed the recommendation of Jbabdi et al. (2015): "Building an accurate picture of white matter pathways requires care, and anatomically informed priors, such as where a pathway can go and where it must not, can provide important constraints for obtaining good results." This is why we decided to use carefully chosen exclusion masks during the streamline extraction process ("tckedit" with 'include' and 'exclude' options). Moreover, we used a whole brain tractography approach and a recommended post-processing step - SIFT (Tournier et al. 2010; Smith et al. 2013) to reduce the signal prediction errors and tractography biases (i.e.: streamlines seeding, white matter volume, reconstruction biases) (Smith et al. 2013, Maier-Hein et al. 2017). Although it is not possible to remove all false positives that could arise from FOD fields, the SIFT algorithm helped us to improve the tractography mainly by removing false positives present in the FOD map after thresholding at the cut-off amplitude of 0.1 . Furthermore, since the extraction of the streamlines of interest was performed from the whole brain tractography, biases related to seed and target volumes were avoided. However, the proportion of associative fiber inputs to the STN coming from the direct and indirect pathways compared to those coming from the hyperdirect pathway remains to be determined. As a whole, we found that the overall direct inputs from the associative cortices to the human STN were very weak and did not allow us to delineate any associative anatomo-functional territory. 
Second, the segmentation of the cortex is not similar from one study to another since boundaries are difficult to define. The attribution of a given cortical area to motor, associative or limbic functions may differ because some cortical areas have multiple functions and others are at the transition between what was considered as motor or executive control. For example, the ventral premotor cortex BA 6 in humans and monkeys has both motor and cognitive functions (for review, Rizzolati et al. 2002). Moreover, there is a smooth gradient between primary motor areas to more complex premotor areas towards pure cognitive areas such as the dorsolateral prefrontal cortex that encodes abstract information to finally make and execute decisions.

Another difficulty for tractography is that the required number of streamlines is unknown. Nevertheless, a greater number of streamlines improves reliability of results by reducing random sampling artefacts and by improving the performance of the SIFT algorithm which can reach a better fitting of diffusion data. We have generated 100 million streamlines to optimize the use of the hardware at our disposal. Generating a greater number of streamlines requires long computation times and high performance machines (for example: generating 100 million streamlines and filtering to 10 million with SIFT for $1.25 \mathrm{~mm}$ iso-voxel data required more than 100 GB of RAM). Nevertheless, our tractography parameters allowed delineation of small anatomo-functional territories of the STN and MSR.

Additionally, our data-driven method relied on the multi-shell tissue response functions provides good reconstruction of the streamlines (Wilkins et al. 2015), and a better performance for the study of long distance connectivity (such as the hyperdirect pathway) compared to global tractography (Sinke et al. 2018). However, it would be interesting to compare results obtained with the most recent tractography approaches such as global tractography. By calibrating the fiber response and by integrating local and global reconstructions, this approach could provide reconstruction of more complex fiber configurations (Christiaens et al. 2015)

\section{Refining DBS targets}

Our data confirm the well-known anatomo-functional organization of the STN, with a posterolateral STN target to treat PD motor symptoms and an anteromedial STN target to treat resistant obsessive-compulsive disorders. However, it is interesting to note that if the posterolateral part of the nucleus is the most widely used target for PD, some surgical teams choose a slightly more anterior target with an even higher efficacy (Benabid et al. 2009; Welter et al. 2014). Indeed, if tremor and rigidity is highly alleviated by posterolateral STN DBS, akinesia, that is not only a pure motor symptom, might better improved with a target position that involves premotor and /or cognitive control. For this reason, we hypothesize that the center of the STN, which integrates 
associative information coming from external pallidal afferences and premotor information coming from cortical projections, should be the location of DBS within the STN to optimally improve the three cardinal symptoms of PD. It is also clear that psychiatric complications of DBS encountered by PD patients especially when the effective electrode contact is positioned anteromedially (Kim et al. 2012; Mallet et al. 2007; Welter et al. 2014) may be explained by the disruption of the limbic STN. On the other hand, DBS of the anteromedial STN for resistant obsessive-compulsive disorders produces beneficial effects obtained with relatively low voltage for the majority of patients in the long term follow up (Chabardes et al. 2013). However, even if the partition in two anatomo-functional territories of STN based on cortical inputs is coherent in all subjects, their boundaries show inter-individual variations that should be taken into account in the future in order to maximize the therapeutic effect of a precise and personalized target.

Our data highlight that the MSR, and especially its medial part, is an important relay of the limbic system that receives dense limbic cortical inputs. This specific area involves the so-called Sano triangle also sometimes referred to as the posterior hypothalamus (Sano 1962). Neurosurgical lesioning of the Sano triangle has been used in the past to treat patients with pathologically violent behavior associated with various psychiatric disorders and who were institutionalized due to treatment-resistance and to the significant risk they present to themselves and others (Sano et al. 1966). Today, DBS of this medial target has been reported to significantly improve pathological violence in treatment-resistant patients (Franzini et al. 2013; Rizzi et al. 2017). It is interesting to note that this target into the MSR is similar to the one used for treating refractory chronic cluster headache with DBS (Leone et al., 2001; Franzini et al. 2003; Akram et al. 2017a). These attacks consist of violent agonizing pain associated with vegetative reactions that could be easily understood by a dysfunction of limbic areas. More recently, the superolateral branch of the medial forebrain bundle was discovered as a possible DBS target to treat resistant depression (Coenen et al. 2011). This bundle known to consist of multiple distinct circuits such as serotonin, norepinephrine, and dopamine systems, passes through the lateral hypothalamus and is connected not only to prefrontal cortex but also to the lateral orbitofrontal region as recently described in humans (Coenen et al. 2018). It is thus possible that DBS of this bundle also stimulates adjacent structures such as the MSR. However, a detailed 3D description of the structural organization of the MSR remains to be provided with its specific connectivity.

\section{Conclusion}


Our data provide evidence that the hyperdirect cortical pathway mainly consists of sensorimotor cortical projections to the posterolateral STN with a descending gradient towards the MSR. Limbic cortical projections also participate in the hyperdirect pathway and specifically innervate the anteromedial STN, with a low density of projections that follow an ascending gradient form the posterolateral STN towards the MSR. The sensorimotor and limbic anatomo-functional territories could be delineated within the STN based on cortical inputs with zones of overlap. In the future, the use of each patient's MRI with high quality DWI should be helpful to refine individual targeting and help to define the best personalized target for functional neurosurgery. 


\section{Funding}

This work was supported by 'Investissements d'avenir' (Investing in the future programme ANR-10-IAIHU-O6). G. Temiz was supported by the "Fondation pour la Recherche" Médicale (FRM) (Project:DIC20161236441) and by Boston Scientific.

Financial disclosures: The authors declare that there are no potential financial conflicts of interest.

Conflict of interest: The authors declare no other conflicts of interest 
Table 1: Mean number of cortical streamlines (\%) with STN and MSR.

\begin{tabular}{|c|c|c|c|c|c|c|}
\hline & \multicolumn{3}{|c|}{ RH } & \multicolumn{3}{|c|}{ LH } \\
\hline & \multicolumn{2}{|c|}{$\begin{array}{l}\text { Mean number of } \\
\text { streamlines (\%) }\end{array}$} & \multirow[t]{2}{*}{$\mathrm{p}$ value } & \multicolumn{2}{|c|}{$\begin{array}{l}\text { Mean number of } \\
\text { streamlines (\%) }\end{array}$} & \multirow[t]{2}{*}{$\mathrm{p}$ value } \\
\hline BA & STN & MSR & & STN & MSR & \\
\hline '1-2-3' & 10.772 & 0.365 & $0.000(* * *)$ & 13.730 & 0.463 & $0.000(* * *)$ \\
\hline$' 4^{\prime}$ & 28.606 & 1.175 & $0.000(* * *)$ & 31.564 & 3.238 & $0.000(* * *)$ \\
\hline$' 6 '$ & 39.977 & 4.876 & $0.000(* * *)$ & 36.655 & 7.433 & $0.000(* * *)$ \\
\hline '8' & 0.578 & 0.723 & 0.210 & 0.893 & 0.933 & 0.530 \\
\hline '5' & 2.183 & 0.101 & $0.000(* * *)$ & 3.514 & 0.284 & $0.000(* * *)$ \\
\hline '7' & 0.6710 & 0.081 & $0.000(* * *)$ & 1.429 & 0.122 & $0.000(* * *)$ \\
\hline '9' & 0.250 & 0.371 & 0.420 & 0.311 & 0.434 & 0.290 \\
\hline '10' & 0.015 & 0.014 & 0.060 & 0.018 & 0.017 & 0.530 \\
\hline '37' & 0.101 & 1.614 & $0.000(* * *)$ & 0.097 & 1.582 & $0.000(* * *)$ \\
\hline '39' & 0.183 & 0.014 & $0.000(* * *)$ & 0.156 & 0.023 & $0.000(* * *)$ \\
\hline '40' & 0.230 & 0.007 & $0.000(* * *)$ & 0.602 & 0.081 & $0.000(* * *)$ \\
\hline '46' & 0.075 & 0.115 & 0.570 & 0.082 & 0.087 & 0.970 \\
\hline$' 47^{\prime}$ & 0.010 & 0.000 & $0.040(*)$ & 0.010 & 0.023 & 0.610 \\
\hline '18' & 0.003 & 0.074 & $0.020(*)$ & 0.026 & 0.046 & 0.420 \\
\hline '19' & 0.116 & 0.108 & $0.020(*)$ & 0.153 & 0.052 & $0.010(* *)$ \\
\hline '20' & 0.057 & 3.782 & $0.000(* * *)$ & 0.056 & 3.244 & $0.000(* * *)$ \\
\hline '21' & 0.049 & 0.027 & 0.120 & 0.005 & 0.006 & 1.000 \\
\hline
\end{tabular}




\begin{tabular}{|c|c|c|c|c|c|c|}
\hline '22' & 0.046 & 0.007 & $0.000(* * *)$ & 0.036 & 0.006 & $0.020\left(^{*}\right)$ \\
\hline '44' & 0.150 & 0.034 & $0.000(* * *)$ & 0.105 & 0.081 & 0.250 \\
\hline '45' & 0.008 & 0.007 & 0.600 & 0.036 & 0.052 & 0.990 \\
\hline '29' & 0.008 & 0.014 & 0.700 & 0.015 & 0.000 & $0.040\left(^{*}\right)$ \\
\hline '38' & 0.003 & 0.027 & 0.290 & 0.000 & 0.041 & $0.010(* *)$ \\
\hline '41' & 0.003 & 0.000 & 0.330 & 0.010 & 0.000 & 0.080 \\
\hline '42' & 0.005 & 0.000 & 0.160 & 0.015 & 0.006 & 0.060 \\
\hline '43' & 0.397 & 0.061 & $0.000(* * *)$ & 0.253 & 0.041 & $0.000(* * *)$ \\
\hline '17' & 0.000 & 0.054 & $0.010(* *)$ & 0.000 & 0.029 & $0.020\left(^{*}\right)$ \\
\hline '11' & 0.003 & 0.000 & 0.330 & 0.000 & 0.000 & - \\
\hline '28' & 0.003 & 0.142 & $0.000(* * *)$ & 0.000 & 0.098 & $0.000(* * *)$ \\
\hline '32' & 0.204 & 0.480 & $0.040(*)$ & 0.385 & 0.550 & $0.010(* *)$ \\
\hline$' 25 '$ & 0.077 & 49.865 & $0.000(* * *)$ & 0.028 & 44.560 & $0.000(* * *)$ \\
\hline '34' & 0.217 & 13.522 & $0.000(* * *)$ & 0.077 & 23.433 & $0.000(* * *)$ \\
\hline '35' & 3.551 & 7.693 & 0.230 & 1.638 & 5.556 & $0.040\left(^{*}\right)$ \\
\hline '30' & 10.462 & 5.403 & $0.040(*)$ & 5.857 & 2.682 & $0.000(* * *)$ \\
\hline '36' & 0.021 & 0.858 & $0.000(* * *)$ & 0.005 & 0.736 & $0.000(* * *)$ \\
\hline '23' & 0.095 & 0.007 & $0.000(* * *)$ & 0.342 & 0.070 & $0.010(* *)$ \\
\hline '26' & 0.018 & 0.000 & $0.010(* *)$ & 0.048 & 0.006 & $0.010(* *)$ \\
\hline '27' & 0.044 & 4.978 & $0.000(* * *)$ & 0.824 & 1.448 & $0.000(* * *)$ \\
\hline '24' & 0 & 0.007 & 0.330 & 0.008 & 0.012 & 0.720 \\
\hline '48' & 0.810 & 3.397 & 0.070 & 1.016 & 2.526 & 0.160 \\
\hline
\end{tabular}


BA, Brodmann area; LH, left hemisphere; RH, right hemisphere. ${ }^{*} \mathrm{p} \leq 0.05 ; * * \mathrm{p} \leq 0.01$; *** $\mathrm{p} \leq 0.001$ (in bold). 


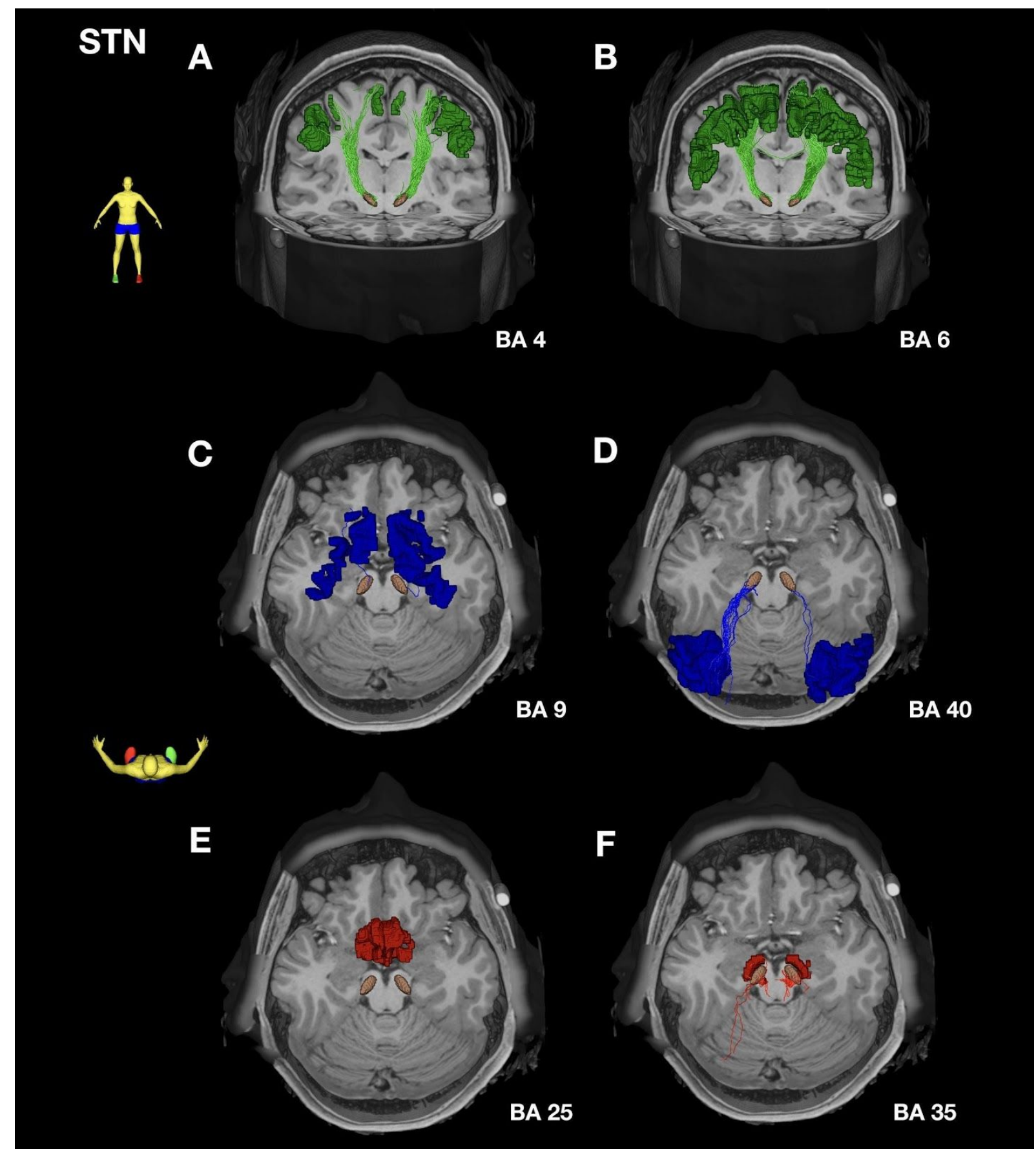

Figure 1: Examples of streamlines connecting cortical areas with the STN in 3D. STN connections with the primary motor cortex BA 4 (A) and premotor cortex BA 6 (B) are represented in green, with the associative prefrontal cortex BA 9 (C) and parietal cortex BA 40 (D) in blue, and with the limbic subgenual cortex BA 25 (E) and perirhinal cortex BA 35 (F) in red. Note that numerous connections from the motor cortical areas were observed. 


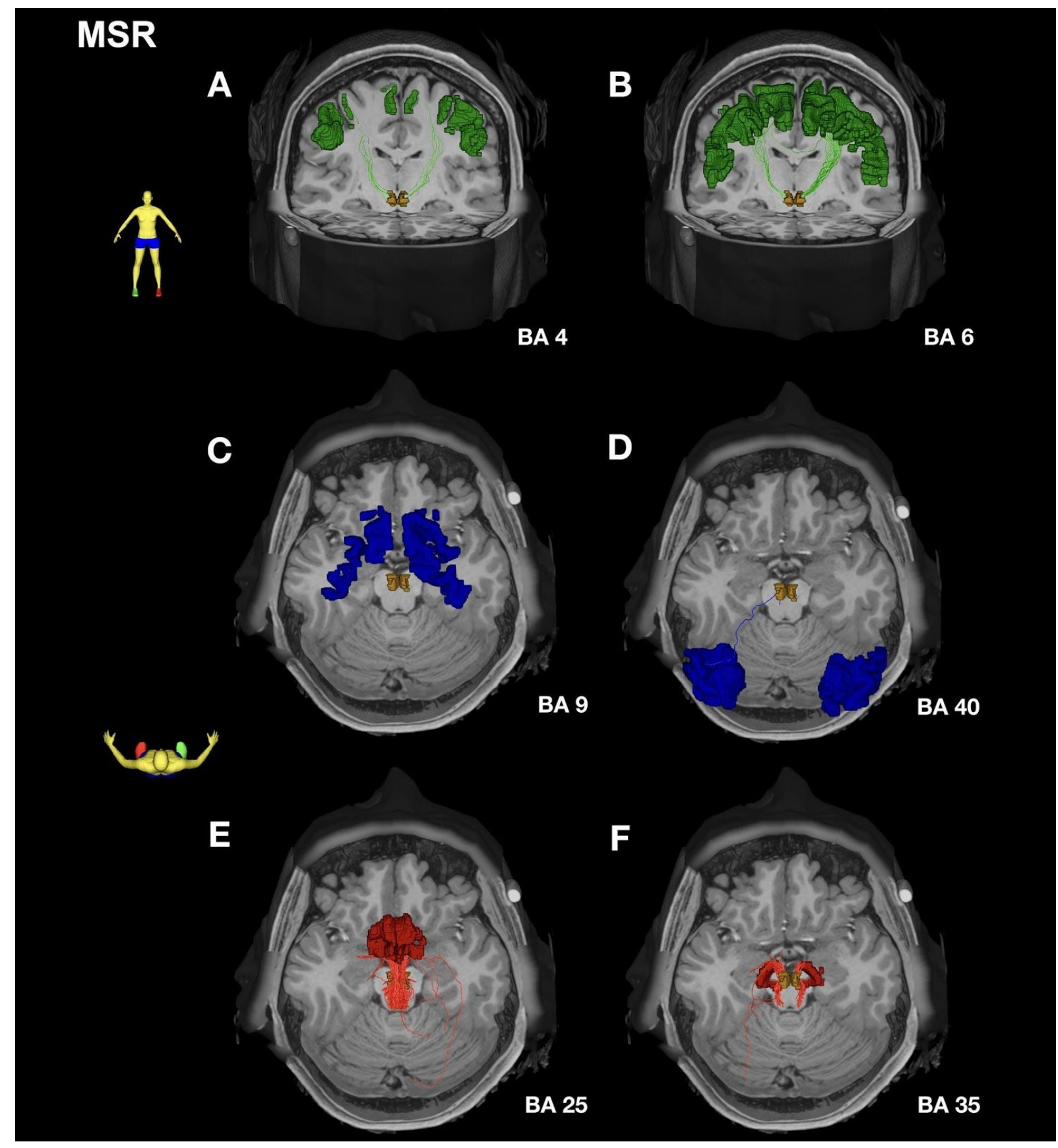

Figure 2: Examples of streamlines connecting cortical areas with the MSR in 3D. MSR connections with the primary motor cortex BA 4 (A) and premotor cortex BA 6 (B) are represented in green, with the associative prefrontal cortex BA 9 (C) and parietal cortex BA 40 (D) in blue, and with the limbic subgenual cortex BA 25 
(E) and perirhinal cortex BA 35 (F) in red. Note that numerous connections from the limbic cortical areas were observed.

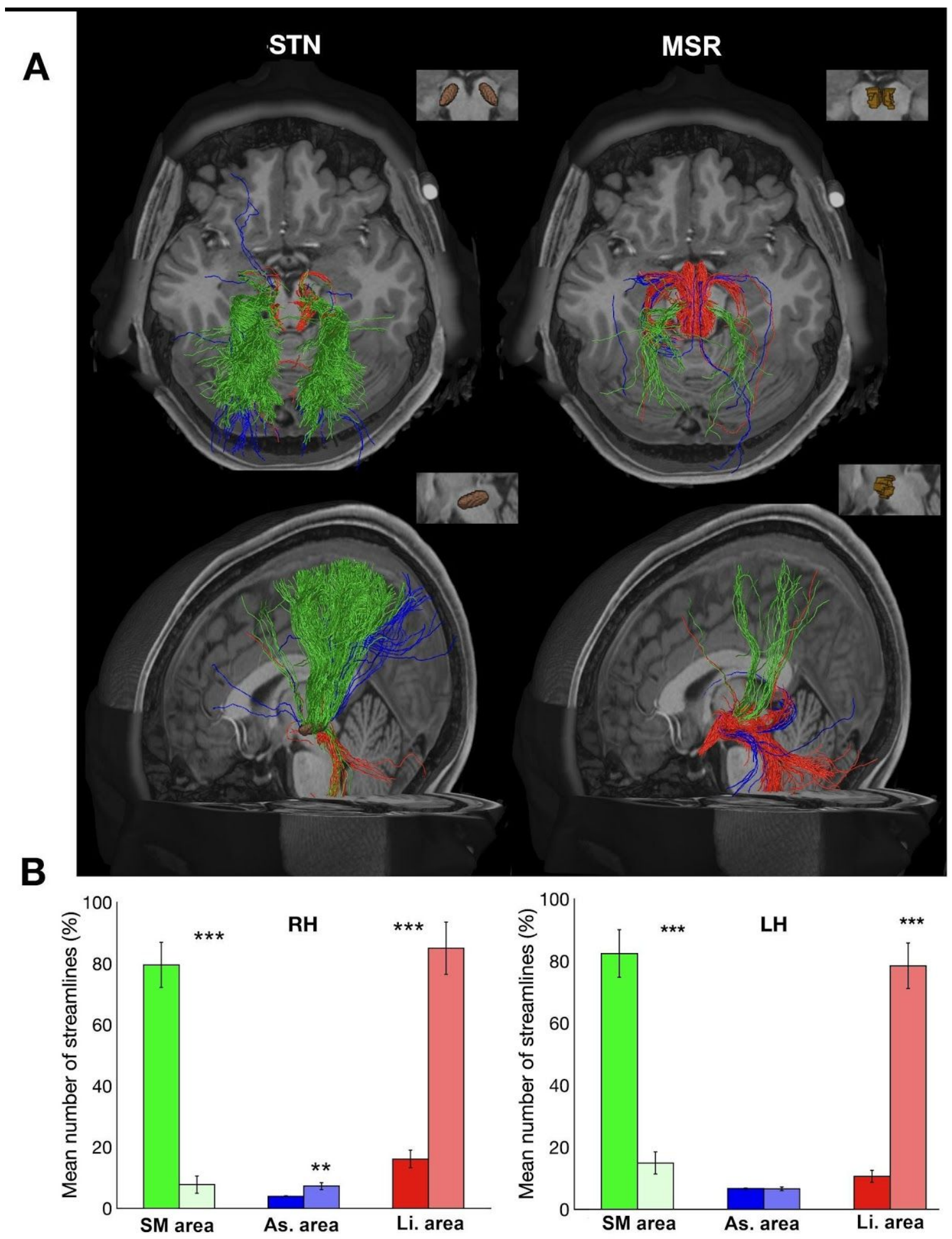


Figure 3: $3 \mathrm{D}$ views of streamlines connecting the overall cortex with the STN and MSR. (A) 3D horizontal view (upper line) and sagittal view (lower line) of the overall sensorimotor (in green), associative (in blue), and limbic (in red) cortical streamlines connected to the STN (left) and the MSR (right). The STN and the MSR masks are illustrated. Note that the STN received mostly from sensorimotor cortical projections whereas the MSR receives mainly from limbic cortical projections. (B) Histogram showing the total mean number of streamlines, expressed as percentage $(\%)$, connecting the cortical areas (sensorimotor, associative, or limbic) to the STN (dark bars) or MSR (bright bars): the mean streamline numbers were averaged over 30 subjects. The error bars represent standard deviations. $* * \mathrm{p} \leq 0.01 ; * * * \mathrm{p} \leq 0.001$

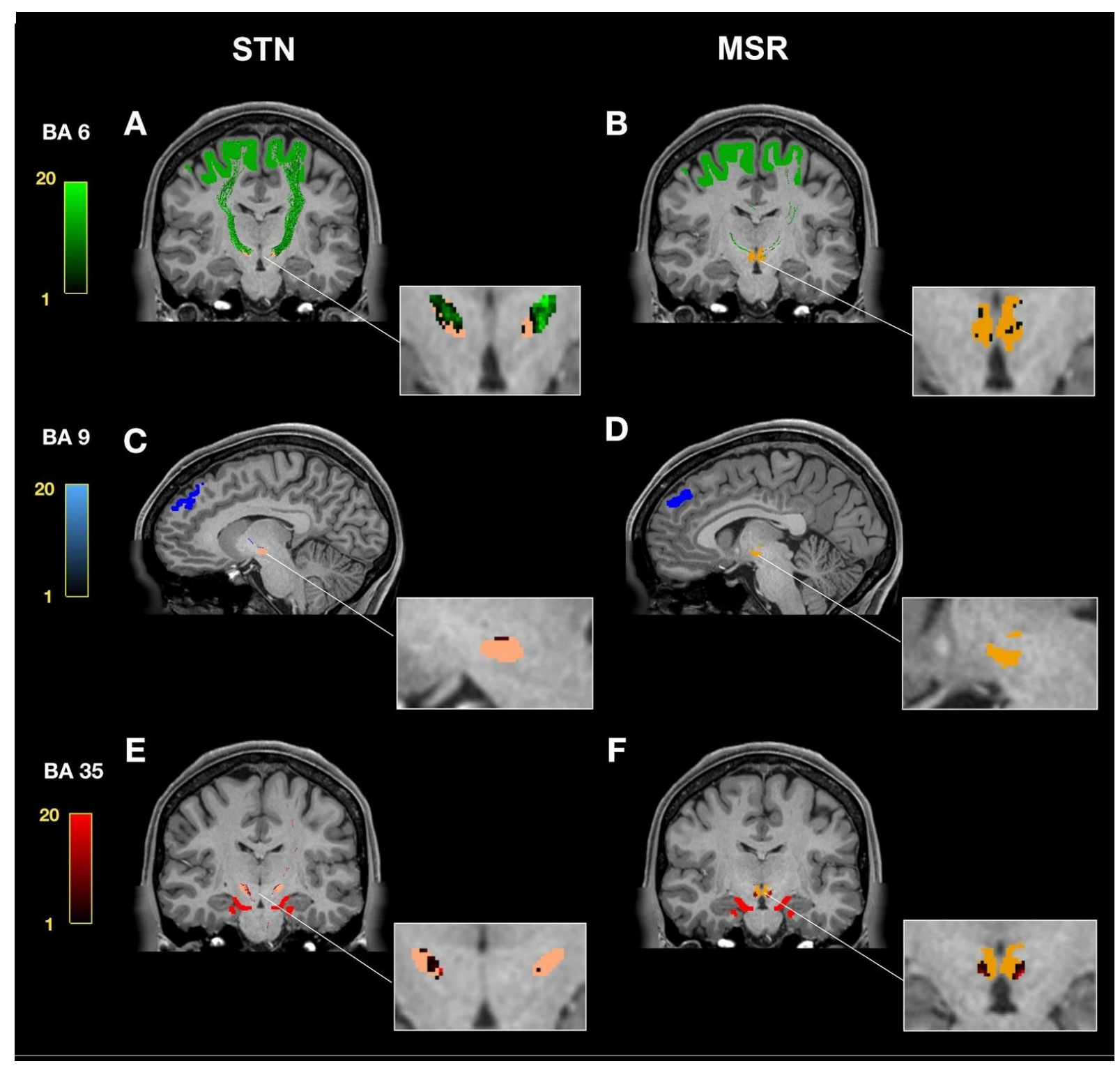


Figure 4: Comparison of the number of cortical streamlines connecting the STN (A, C, E) and the MSR (B, D, F). Streamlines and corresponding density maps were illustrated for the premotor cortex BA 6 (A, B, coronal slices), the associative prefrontal cortex BA 9 (C, D, sagittal slices), and the limbic perirhinal cortex BA 35 (E, F, coronal slices). Color-bars $[1 ; 20]$ are shown for the connectivity with BA 6 (in shades of green), BA 9 (in shades of blue) and BA 35 (in shades of red), and the corresponding voxels are shown on the STN (flesh) and MSR (orange) masks.

A

Mean number of streamlines (\%)

A
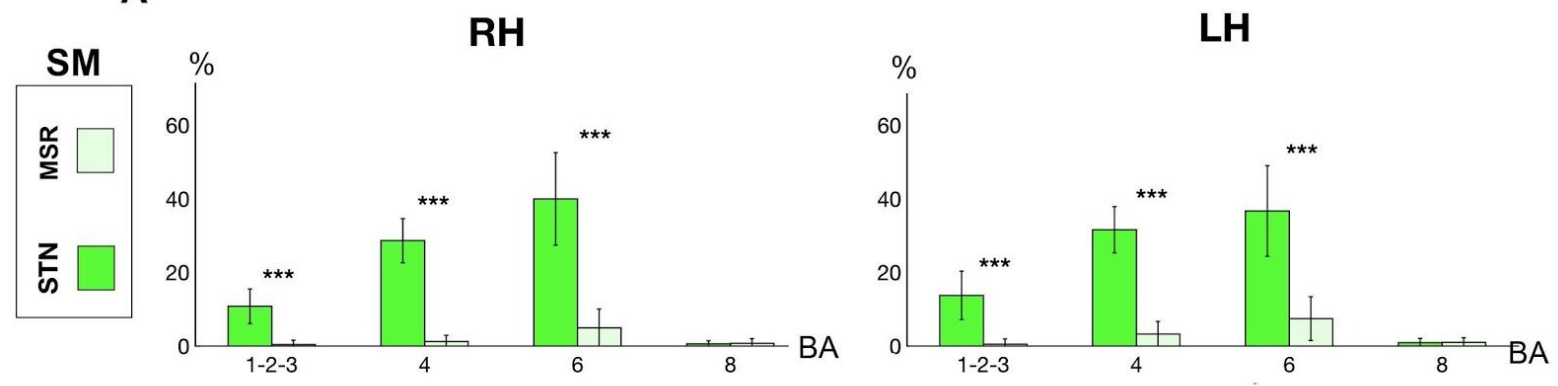

B

As.
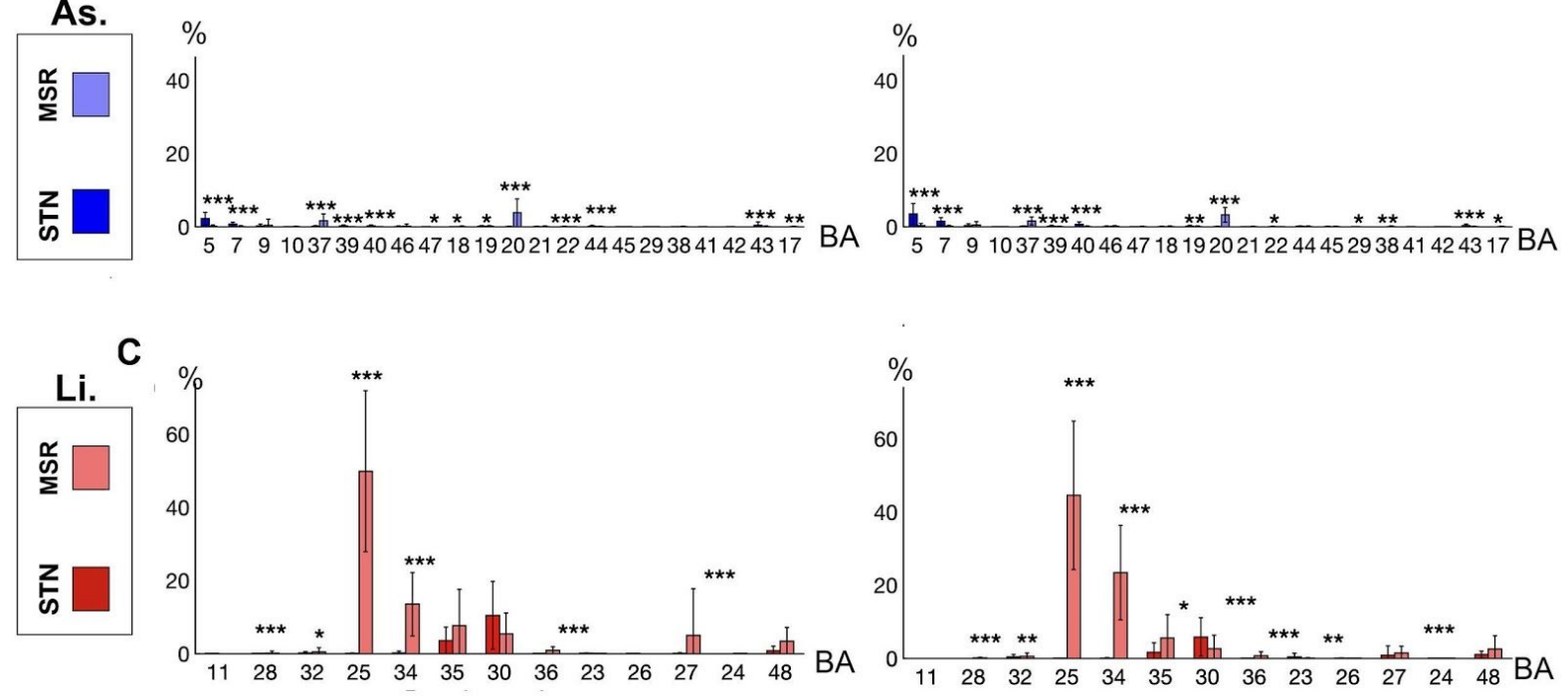

Figure 5: Histograms showing connectivity of the STN and MSR to different cortical areas. The percentages of mean streamline numbers connecting the different sensorimotor (SM in green), associative (As. in blue) and limbic (Li. in red) cortical areas to the STN (dark bars) and to the MSR (bright bars) are shown for the right hemisphere (RH) and the left hemisphere (LH). Percentage values were averaged across 30 subjects. Sensorimotor cortical connectivity is higher with the STN than with the MSR, whereas the limbic cortical areas 
are highly connected to the MSR than to the STN. The associative cortical areas are weakly connected to both the STN and the MSR. * $\mathrm{p} \leq 0.05 ; * * \mathrm{p} \leq 0.01 ; * * \mathrm{p} \leq 0.001$

\section{A}
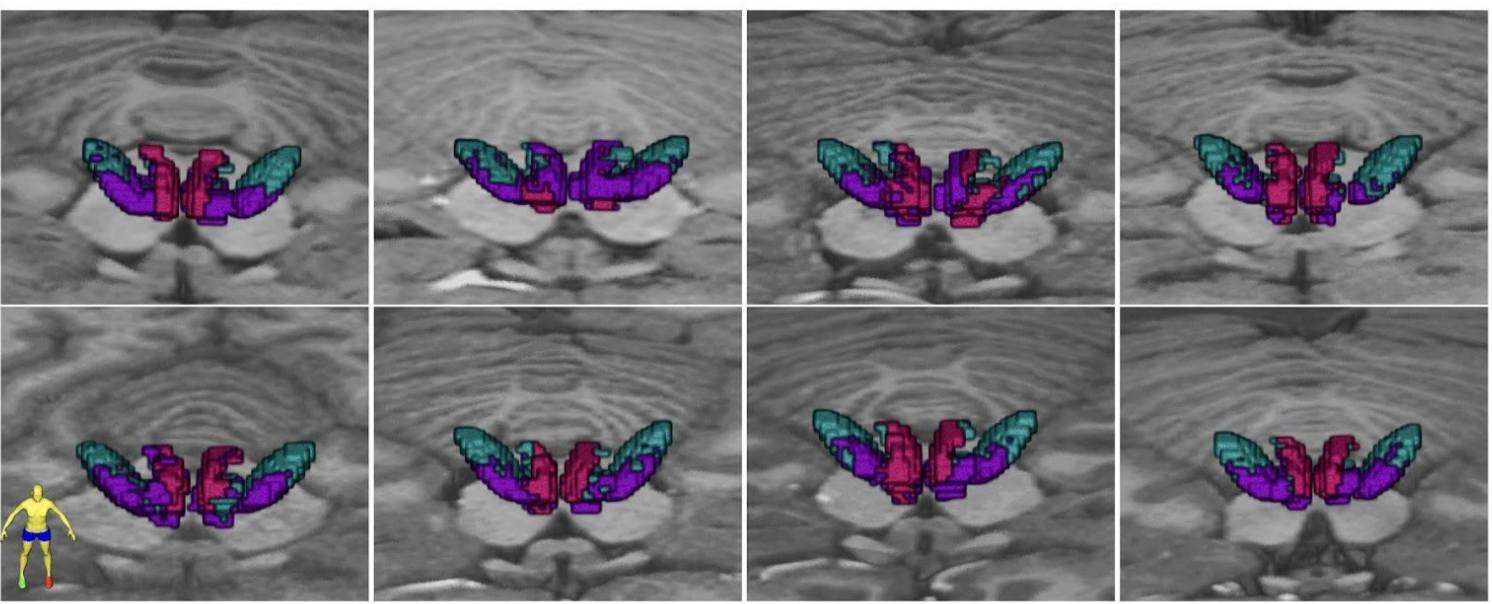

Cluster 1

Cluster 2

Cluster 3

B
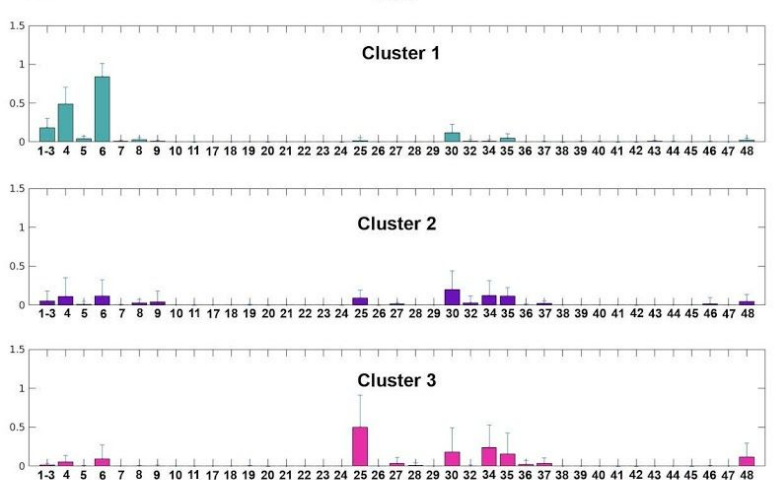

LH

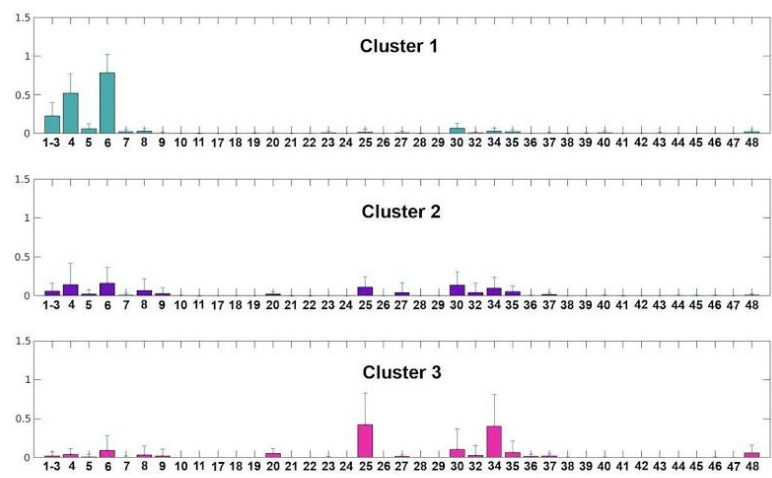

Figure 6: Spatial distribution of the three clusters in the STN area in 8 subjects. (A) Cluster 1 (dark cyan) is located posterolaterally in the STN, cluster 2 (purple) is located anteromedially in the STN and in the MSR, and cluster 3 (pink) is located in the medial part of the MSR. (B) Histograms showing global mean connectivity profiles of cluster 1 (upper line, dark cyan bars), cluster 2 (intermediate lines, purple bars), and cluster 3 (lower lines, pink bars) averaged over 30 subjects for the right hemisphere (RH) and the left hemisphere (LH). 


\section{References}

Accolla EA, Dukart J, Helms G, Weiskopf N, Kherif F, Lutti A, Chowdhury R, Hetzer S, Haynes JR, Kühn AA, Draganski B (2014) Brain tissue properties differentiate between motor and limbic basal ganglia circuits. Hum Brain Mapp 35 (10):5083-5092

Afsharpour S (1985) Topographical projections of the cerebral cortex to the subthalamic nucleus. J Comp Neurol 236:14-28

Akram H, Miller S, Lagrata S, Hariz M, Ashburner J, Behrens T, Matharu M, Zrinzo L (2017a) Optimal deep brain stimulation site and target connectivity for chronic cluster headache. Neurology 89(20):2083-2091. doi: 10.1212/WNL.0000000000004646

Akram H, Sotiropoulos SN, Jbabdi S, Georgiev D, Mahlknecht P, Hyam J, Foltynie T, Limousin P, De Vita E, Jahanshahi M, Hariz M, Ashburner J, Behrens T, Zrinzo L (2017b). Subthalamic deep brain stimulation sweet spots and hyperdirect cortical connectivity in Parkinson's disease. NeuroImage 158:332-345. doi: 10.1016/j.neuroimage.2017b.07.012.

Alkemade A, Forstmann BU (2014) Do we need to revise the tripartite subdivision hypothesis of the human subthalamic nucleus (STN)? NeuroImage 95:326-329

Andersson JL, Sotiropoulos SN (2015) Non-parametric representation and prediction of single- and multi-shell diffusion-weighted MRI data using Gaussian processes. NeuroImage 122:166-176

Aravamuthan BR, Muthusamy KA, Stein JF, Aziz TZ, Johansen-Berg H (2007) Topography of cortical and subcortical connections of the human pedunculopontine and subthalamic nuclei. NeuroImage 37:694-705. doi:10.1016/j.neuroimage.2007.05.050

Aron AR, Behrens TE, Smith S, Frank MJ, Poldrack RA (2007) Triangulating a cognitive control network using diffusion-weighted magnetic resonance imaging (MRI) and functional MRI. J Neurosci 27 (14):3743-3752

Avecillas-Chasin JM, Rascón-Ramírez F (2016) Tractographical model of the cortico-basal ganglia and corticothalamic connections: Improving Our Understanding of Deep Brain Stimulation. Clin Anat 29(4):481-492

Bardinet E, Bhattacharjee M, Dormont D, Pidoux B, Malandain G, Schüpbach M, Ayache N, Cornu P, Agid Y, Yelnik J (2009) A three-dimensional histological atlas of the human basal ganglia. II. Atlas deformation strategy and evaluation in deep brain stimulation for Parkinson disease. J Neurosurg 110:208-219. http://dx.doi.org/10.3171/2008.3.17469 
Bejjani BP, Houeto JL, Hariz M, Yelnik J, Mesnage V, Bonnet AM, Pidoux B, Dormont D, Cornu P, Agid Y (2002) Aggressive behavior induced by intraoperative stimulation in the triangle of Sano. Neurology 59(9):1425-1427

Benabid AL, Chabardes S, Mitrofanis J, Pollak P (2000) Deep brain stimulation of the subthalamic nucleus for the treatment of Parkinson's disease. Lancet Neurol 8(1):67-81. doi: 10.1016/S1474-4422(08)70291-6

Brunenberg EJ, Moeskops P, Backes WH, Pollo C, Cammoun L, Vilanova A, Janssen ML, Visser-Vandewalle VE, ter Haar Romeny BM, Thiran JP, Platel B (2012) Structural and resting state functional connectivity of the subthalamic nucleus: identification of motor STN parts and the hyperdirect pathway. PLoS One 7(6, :e39061)

Calamante F, Tournier JD, Jackson GD, Connelly A (2010) Track-density imaging (TDI): Super-resolution white matter imaging using whole-brain track-density mapping. NeuroImage 53:1233-1243

Canteras NS, Shammah-Lagnado SJ, Silva BA, Ricardo JA (1990) Afferent connections of the subthalamic nucleus: a combined retrograde and anterograde horseradish peroxidase study in the rat. Brain Res $513: 43-59$

Chabardès S, Polosan M, Krack P, Bastin J, Krainik A, David O, Bougerol T, Benabid AL (2013) Deep brain stimulation for obsessive-compulsive disorder: subthalamic nucleus target. World Neurosurg 80(3-4):S31.e1-8. doi: 10.1016/j.wneu.2012.03.010

Christiaens D, Reiser M, Dhollander T, Sunaert S, Suetens P and Maes F. Global (2015) tractography of multi shell diffusion weighted imaging data using a multi-tissue model. NeuroImage, 123:89-101

Coenen VA, Schlaepfer TE, Maedler B, Panksepp J (2011) Cross-species affective functions of the medial forebrain bundle-implications for the treatment of affective pain and depression in humans. Neurosci Biobehav Rev 35(9):1971-1981. doi: 10.1016/j.neubiorev.2010.12.009

Coenen VA, Schumacher LV, Kaller C, Schlaepfer TE, Reinacher PC, Egger K, Urbach H, Reisert M (2018) The anatomy of the human medial forebrain bundle: Ventral tegmental area connections to reward-associated subcortical and frontal lobe regions. Neuroimage Clin 18:770-783. doi: 10.1016/j.nicl.2018.03.019

Coudé D, Parent A, Parent M (2018) Single-axon tracing of the corticosubthalamic hyperdirect pathway in primates. Brain Struct Funct 223(9):3959-3973. doi: 10.1007/s00429-018-1726-X 
Crossman AR, Sambrook MA, Jackson A (1984) Experimental hemichorea / hemiballismus in the monkey. Studies on the intracerebral site of action in a drug-induced dyskinesia. Brain 107 (Pt 2):579-596

Dhollander T, Raffelt D, Connelly A (2016) Unsupervised 3-tissue response function estimation from single-shell or multi-shell diffusion MR data without a co-registered T1 image. ISMRM Workshop on Breaking the Barriers of Diffusion MRI, 5

Forel A (1877) Untersuchungen über die Haubenregion und ihre oberen Verknüpfungen im Gehirne des Menschen und einiger Säugethiere, mit Beiträgen zu den Methoden der Gehirnuntersuchung. Archiv für Psychiatrie und Nervenkrankheiten. Springer-Verlag 7(3):393-495

François C, Grabli D, McCairn K, Jan C, Karachi C, Hirsch EC, Féger J, Tremblay L (2004) Behavioural disorders induced by external globus pallidus dysfunction in primates II. Anatomical study. Brain 127(Pt 9):2055-2070

Franzini A, Broggi G, Cordella R, Dones I, Messina G (2013) Deep-brain stimulation for aggressive and disruptive behavior. World Neurosurg 80(3-4):S29

Franzini A, Ferroli P, Leone M, Broggi G (2003) Stimulation of the posterior hypothalamus for treatment of chronic intractable cluster headaches: first reported series. Neurosurgery 52(5):1095-1099

Gan G, Ma C, Wu J (2007) Data Clustering: Theory, Algorithms, and Applications. Vol 20 Siam

Glasser MF, Sotiropoulos SN, Wilson JA, Coalson TS, Fischl B, Andersson JL, Xu J, Jbabdi S, Webster M, Polimeni JR, Van Essen DC, Jenkinson M (2013) The minimal preprocessing pipelines for the Human Connectome Project. Neuroimage 80:105-124. doi:10.1016/j.neuroimage.2013.04.127

Goto M, Swanson LW (2004) Axonal projections from the parasubthalamic nucleus. J Comp Neurol 169 (4):581-607

Haber SN, Lynd-Balta E, Mitchell SJ (1993) The organization of the descending ventral pallidal projections in the monkey. J Comp Neurol 329(1):111-128

Haber SN, Fudge JL, McFarland NR (2000) Striatonigrostriatal pathways in primates form an ascending spiral from the shell to the dorsolateral striatum. J Neurosci 20(6):2369-2382.

Hammond C, Yelnik J (1983) Intracellular labelling of rat subthalamic neurones with horseradish peroxidase: computer analysis of dendrites and characterization of axon arborization. Neuroscience 8(4):781-790

Hartmann-von Monakow K, Akert K, Künzle H (1978) Projections of the precentral motor cortex and other cortical areas of the frontal lobe to the subthalamic nucleus in the monkey. Exp Brain Res 33:395-403 
Haynes W, Haber S (2013) The organization of prefrontal subthalamic inputs in primates provides an anatomical substrate for both functional specificity and integration: implications for basal ganglia models and deep brain stimulation. J Neuroscience 33:4804-4814

Jbabdi S, Sotiropoulos SN, Haber SN, Van Essen DC, Behrens TE (2015) Measuring macroscopic brain connections in vivo. Nat Neurosci 18(11):1546-55. doi: 10.1038/nn.4134. Epub 2015 Oct 27

Jeurissen B, Tournier JD, Dhollander T, Connelly A, Sijbers J (2014) Multi-tissue constrained spherical deconvolution for improved analysis of multi-shell diffusion MRI data. Neuroimage 103:411-426

Jürgens U (1984) The efferent and afferent connections of the supplementary motor area. Brain Res 300:63-81

Karachi C, Yelnik J, Tandé D, Tremblay L, Hirsch E, François C (2005) The pallido- subthalamic projection: an anatomical substrate for nonmotor functions of the subthalamic nucleus in primates. Mov Disord 20 (2):172-180

Karachi C, Grabli D, Baup N, Mounayar S, Tandé D, François C, Hirsch EC (2009) Dysfunction of the subthalamic nucleus induces behavioral and movement disorders in monkeys. Mov Disord 24(8):1183-1192. doi: 10.1002/mds.22547

Kim JS, Kim HJ, Lee JY, Kim JM, Yun JY, Jeon BS (2012) Hypomania induced by subthalamic nucleus stimulation in a Parkinson's disease patient: does it suggest a dysfunction of the limbic circuit? J Mov Disord 5(1):14-17. doi: 10.14802/jmd.12004

Kunzle H (1975) Bilateral projections from precentral motor cortex to the putamen and other parts of the basal ganglia. An autoradiographic study in Macaca fascicularis. Brain Res 88:195-209

Künzle H (1978) An autoradiographic analysis of the efferent connections from premotor and adjacent prefrontal regions (areas 6 and 9) in Macaca fascicularis. Brain Behav Evol 15:185-234

Künzle H, Akert K (1977) Efferent connections of cortical, area 8 (frontal eye field) in Macaca fascicularis. A reinvestigation using the autoradiographic technique. J Comp Neurol 173:147-164

Lambert C, Zrinzo L, Nagy Z, Lutti A, Hariz M, Foltynie T, Draganski B, Ashburner J, Frackowiak R (2015) Do we need to revise the tripartite subdivision hypothesis of the human subthalamic nucleus (STN)? Response to Alkemade and Forstmann. Neuroimage 110:1-2. doi: 10.1016/j.neuroimage.2015.01.038

Lambert C, Zrinzo L, Nagy Z, Lutti A, Hariz M, Foltynie T, Draganski B, Ashburner J, Frackowiak R (2012) Confirmation of functional zones within the human subthalamic nucleus: Patterns of connectivity and sub-parcellation using diffusion weighted imaging. Neuroimage 63-94. 
doi:10.1016/j.neuroimage.2011.11.082

Leone M, Franzini A, Bussone G (2001) Stereotactic stimulation of posterior hypothalamic gray matter in a patient with intractable cluster headache. N Engl J Med 345(19):1428-1429

Limousin P, Krack P, Pollak P, Benazzouz A, Ardouin C, Hoffman D, Benabid AL (1998) Electrical stimulation of the subthalamic nucleus in advanced Parkinson's disease. N Engl J Med 339:1105-1111

Maier-Hein KH, Neher PF, Houde JC, Côté MA, Garyfallidis E, Zhong J, Chamberland M, [...], Descoteaux M (2017) The challenge of mapping the human connectome based on diffusion tractography. Nature Communications 8:1349. doi: 10.1038/s41467-017-01285-x.

Mallet L, Polosan M, Jaafari N, Baup N, Welter ML, Fontaine D, du Montcel ST, Yelnik J, Chéreau I, Arbus C, Raoul S, Aouizerate B, Damier P, Chabardès S, Czernecki V, Ardouin C, Krebs MO, Bardinet E, Chaynes P, Burbaud P, Cornu P, Derost P, Bougerol T, Bataille B, Mattei V, Dormont D, Devaux B, Vérin M, Houeto JL, Pollak P, Benabid AL, Agid Y, Krack P, Millet B, Pelissolo A; STOC Study Group (2008) Subthalamic nucleus stimulation in severe obsessive-compulsive disorder. $\mathrm{N}$ Engl J Med $359(20): 2121-2134$

Mallet L, Schüpbach M, N'Diaye K, Remy P, Bardinet E, Czernecki V, Welter ML, Pelissolo A, Ruberg M, Agid Y, Yelnik J (2007) Stimulation of subterritories of the subthalamic nucleus reveals its role in the integration of the emotional and motor aspects of behavior. Proc Natl Acad Sci U S A. 104(25), 10661-10666

Maurice N, Deniau JM, Glowinski J, Thierry A (1998) Relationships between the prefrontal cortex and the basal ganglia in the rat: physiology of the corticosubthalamic circuits. J Neurosci 18(22):9539-9546

Mulder MJ, Boekel W, Ratcliff R, Forstmann BU (2014) Cortico-subthalamic connection predicts individual differences in value-driven choice bias Brain Struct Funct 219(4):1239-1249

Nambu A, Takada M, Inase M, Tokuno H (1996) Dual somatotopical representations in the primate subthalamic nucleus: evidence for ordered but reversed body-map transformations from the primary motor cortex and the supplementary motor area. J Neurosci 16 (8):2671-2683

Nambu A, Tokuno H, Inase M, Takada M (1997) Corticosubthalamic input zones from forelimb representations of the dorsal and ventral divisions of the premotor cortex in the macaque monkey: comparison with the input zones from the primary motor cortex and the supplementary motor area. Neurosci Lett 239:13-16 
Neudorfer C, Maarouf M (2018) Neuroanatomical background and functional considerations for stereotactic interventions in the $\mathrm{H}$ fields of Forel. Brain Struct Funct 223(1):17-30. doi: 10.1007/s00429-017-1570-4

Parent A, Hazrati LN (1995) Functional anatomy of the basal ganglia. II. The place of subthalamic nucleus and external pallidum in basal ganglia circuitry. Brain Res Rev 20 (1):128-154

Petersen MV, Lund TE, Sunde N, Frandsen J, Rosendal F, Juul N, Østergaard K (2016) Probabilistic versus deterministic tractography for delineation of the cortico-subthalamic hyperdirect pathway in patients with Parkinson disease selected for deep brain stimulation. J Neurosurg 126(5):1657-1668

Rizzi M, Trezza A, Messina G, De Benedictis A, Franzini A, Marras CE (2017) Exploring the brain through posterior hypothalamus surgery for aggressive behavior. Neurosurg Focus 43(3):E14. doi: 10.3171/2017.6.FOCUS17231.

Rizzolatti G, Fogassi L, Gallese V (2002) Motor and cognitive functions of the ventral premotor cortex. Curr Opin Neurobiol 12(2):149-154

Sano K (1962) Sedative neurosurgery with special reference to posteromedial hypothalamotomy. Neurol Me Chi (Tokyo) 4:112-142

Sano K, Mayanagi Y, Sekino H, Ogashiwa M, Ishijima B (1970) Results of stimulation and destruction of the posterior hypothalamus in man. J Neurosurg 33(6):689-707

Sano K, Yoshioka M, Ogashiwa M, Ishijima B, Ohye C (1966) Postero-medial hypothalamotomy in the treatment of aggressive behaviors. Confinia neurol 27:164-167

Sébille SB, Belaid H, Philippe AC, André A, Lau B, François C, Karachi C, Bardinet E (2017) Anatomical evidence for functional diversity in the mesencephalic locomotor region of primates. Neuroimage 147:66-78. doi: 10.1016/j.neuroimage.2016.12.011

Shink E, Bevan MD, Bolam JP, Smith Y (1996) The subthalamic nucleus and the external pallidum: Two tightly interconnected structures that control the output of the basal ganglia in the monkey. Neuroscience $73: 335-357$

Shook BL, Schlag-Rey M, Schlag J (1991) Primate supplementary eye field. II. Comparative aspects of connections with the thalamus, corpus striatum, and related forebrain nuclei. J Comp Neurol 307:562-583

Sinke M, Otte W, Christiaens D, Schmitt O, Leemans A, Toorn A, Sarabdjitsingh A, Joels M, Dijkhuizen R (2018) Diffusion MRI-based cortical connectome reconstruction: dependency on tractography procedures and neuroanatomical characteristics. Brain Struct. Func. 223(5) :2269-2285 
Smith RE, Tournier JD, Calamante E, Connelly A (2013) SIFT: Spherical-deconvolution informed filtering of tractograms. NeuroImage 67:298-312

Sotiropoulos SN, Jbabdi S, Xu J, Andersson JL, Moeller S, Auerbach EJ, Glasser MF, Hernandez M, Sapiro G, Jenkinson M, Feinberg DA, Yacoub E, Lenglet C, Van Essen DC, Ugurbil K, Behrens TEJ (2013) Advances in diffusion MRI acquisition and processing in the Human Connectome Project. Neuroimage 80:125-143. doi:10.1016/j.neuroimage.2013.05.057

Takada M, Tokuno H, Hamada I, Inase M, Ito Y, Imanishi M, Hasegawa N, Akazawa T, Hatanaka N, Nambu A (2001) Organization of inputs from cingulate motor areas to basal ganglia in macaque monkey. Eur J Neurosci 14(10):1633-1650.

Tournier JD, Calamante F, Connelly A (2010) Improved probabilistic streamlines tractography by 2nd order integration over fibre orientation distributions. Proc Intl Soc Mag Reson Med (ISMRM) 18, p. 1670

Tournier JD, Calamante F, Connelly A (2012) MRtrix: diffusion tractography in crossing fiber regions. Int J Imaging Syst Technol 22:53-66. http://dx.doi.org/ 10.1002/ima.22005.

Ugurlu D, Firat Z, Ture U, Unal G (2018) Neighborhood resolved fiber orientation distributions (NRFOD) in automatic labeling of white matter fiber pathways. Medical Image Analysis 46:130-145

Van Essen DC, Smith SM, Barch DM, Behrens TEJ, Yacoub E, Ugurbil K (2013) The WU-Minn Human Connectome Project: An overview. Neuroimage 80:62-79. doi:10.1016/j.neuroimage.2013.05.041

Veazey RB, Amaral DG, Cowan WM (1982) The morphology and connections of the posterior hypothalamus in the cynomolgus monkey (Macaca fascicularis). II. Efferent connections. J Comp Neurol 207(2):135-156

Wang L, Mruczek EB, Arcaro MJ, Kastner S (2018) Probabilistic maps of visual topography in human cortex. Cerebral Cortex 25:3911-3931

Welter ML, Schüpbach M, Czernecki V, Karachi C, Fernandez-Vidal S, Golmard JL, Serra G, Navarro S, Welaratne A, Hartmann A, Mesnage V, Pineau F, Cornu P, Pidoux B, Worbe Y, Zikos P, Grabli D, Galanaud D, Bonnet AM, Belaid H, Dormont D, Vidailhet M, Mallet, L, Houeto JL, Bardinet E, Yelnik J, Agid Y (2014) Optimal target localization for subthalamic stimulation in patients with Parkinson disease. Neurology 82(15):1352-1361

Wilkins B, Lee N, Gajawelli N, Law M, Leporé N (2015) Fiber estimation and tractography in diffusion MRI: development of simulated brain images and comparison of multi-fiber analysis methods at clinical b-values. Neuroimage 109:341-356 


\section{Supplementary material}

\section{Supplementary Methods}

\section{Image acquisition and preprocessing}

All HCP subjects underwent imaging on a 3.0 Tesla Skyra scanner (Siemens Medical Solutions, Erlangen, Germany) with a customized gradient set, using a 32-channel RF head coil. T1-weighted images were acquired with an isotropic spatial resolution of $0.7 \mathrm{~mm}$ and a total scan time of 7 minutes and 40 seconds. DWI were acquired using a single-shot 2D spin-echo multiband Echo Planar Imaging (EPI) sequence. Each DWI dataset consisted of 3 shells which were acquired twice with reversed phase encoding, defined with b-values of 1000, 2000 and $3000 \mathrm{~s} / \mathrm{m}^{2}, 90$ directions per shell, isotropic spatial resolution of $1.25 \mathrm{~mm}$ and a total scan time of 58 minutes. All HCP DWI datasets were pre-processed for intensity normalization, eddy current, patient motion and EPI distortion correction and co-registered to the anatomical scans (Glasser et al. 2013; Sotiropoulos et al. 2013; Andersson and Sotiropoulos 2015).

\section{Segmentation of regions of interest}

To identify the basal ganglia, we used our in-house 3D histological and deformable atlas (YeB atlas) (Bardinet et al. 2009). In order to segment the MSR, a template was first built by GT from the $30 \mathrm{HCP}$ subjects that were already normalized in the Montreal 
Neurological Institute (MNI) space using the mean T1- and T2-weighted images T1 and T2 images (all HCP data are provided with MNI spatial normalization matrices). Two anatomical experts $(\mathrm{CK}, \mathrm{CF})$ then together manually segmented the right and left MSR on this template. Segmentation was based on the visualization of surrounding structures (mammillo-thalamic tract, zona incerta, substantia nigra, STN) already segmented in the YeB Atlas. Anatomical limits were arbitrary decided since this medial STN region belongs to the reticular formation without clear-cut boundaries. The template was then superposed on the native space of our 30 subjects by applying provided MNI spatial normalization matrices (normalization matrices were provided with HCP data), using FSL (applywarp). Anatomical experts carefully verified the delineation of the MSR and STN on each side of each subject and manual corrections of the MSR were performed if needed.

Cortical areas were segmented using the Brodmann atlas included in the Mricro software (https://www.mccauslandcenter.sc.edu/crnl/mricro) projected to the native space of our subjects. Cortical areas were intersected with the GM/WM boundary (computed with the Freesurfer recon-all pipeline), and dilated (with FSL, kernel size: $3 \times 3 \times 3 \mathrm{~mm}$ ). This resulted in individual Brodmann areas, well adapted to structural connectivity analyses. The cortical areas include 39 cortical masks (each mask corresponding to one or several Brodmann areas (BA)) which were classified into three groups based on their function: sensorimotor cortical areas (BA 1-3, 4, 6, 8), associative areas (BA 5, 7, 9, 10, 17-22, 29, 37-47) and limbic areas (BA 11, 23-28, 30-36, 48). In the cortical atlas that we used, BA 48 corresponds to the insular cortices BA 12-16. We arbitrary chose to group the primary sensory cortical areas in the associative areas group. In this way, the whole cortex was taken into account for our analyses. 
However, it is important to interpret these results cautiously because some cortical areas have both motor and cognitive, or cognitive and limbic functions.

\section{Hierarchical Agglomerative Classification}

Sub-parcellation of the STN and the MSR was then generated by hierarchical agglomerative clustering using Ward's method (Gan et al. 2007) employing euclidean distance as the similarity measure, in the subject's native space. This clustering method has been previously used successfully by Lambert et al. (2012).

A matrix containing the voxel connectivity profiles was generated based on the track-density maps for each hemisphere of each subject. Thus, all matrices contained 39 columns (corresponding to the 39 cortical masks and derived 39 track-density maps). The number of rows varied, depending on the number of the voxels in the STN and MSR. Each row, representing the voxel connectivity profile, was normalized by its maximum track-density value, to avoid the scaling effect on the similarity measurement. Therefore, normalized data fell within the interval $[0,1]$. Clustering consisted in an iterative algorithm that attempts to minimize the sum of squares error at each stage and classify similar voxels through their normalized connectivity profiles, creating a hierarchical cluster tree. The clustering algorithm was run in MATLAB (2016b) with the linkage function.

The hierarchical dendrogram was cut at a height corresponding to three clusters, based on the three anatomo-functional territories identified from the pallido-subthalamic projections (Haber et al. 1993; Shink et al. 1996; Francois et al. 2004; Karachi et al. 2005). Clustering was performed for each subject in its native space. The final step consisted of the 
interpretation of these unsupervised clusters in terms of cortical connectivity. A functional label of these clusters was attributed according to their specific cortical connectivity profile.

\section{Supplementary Figure Legend}
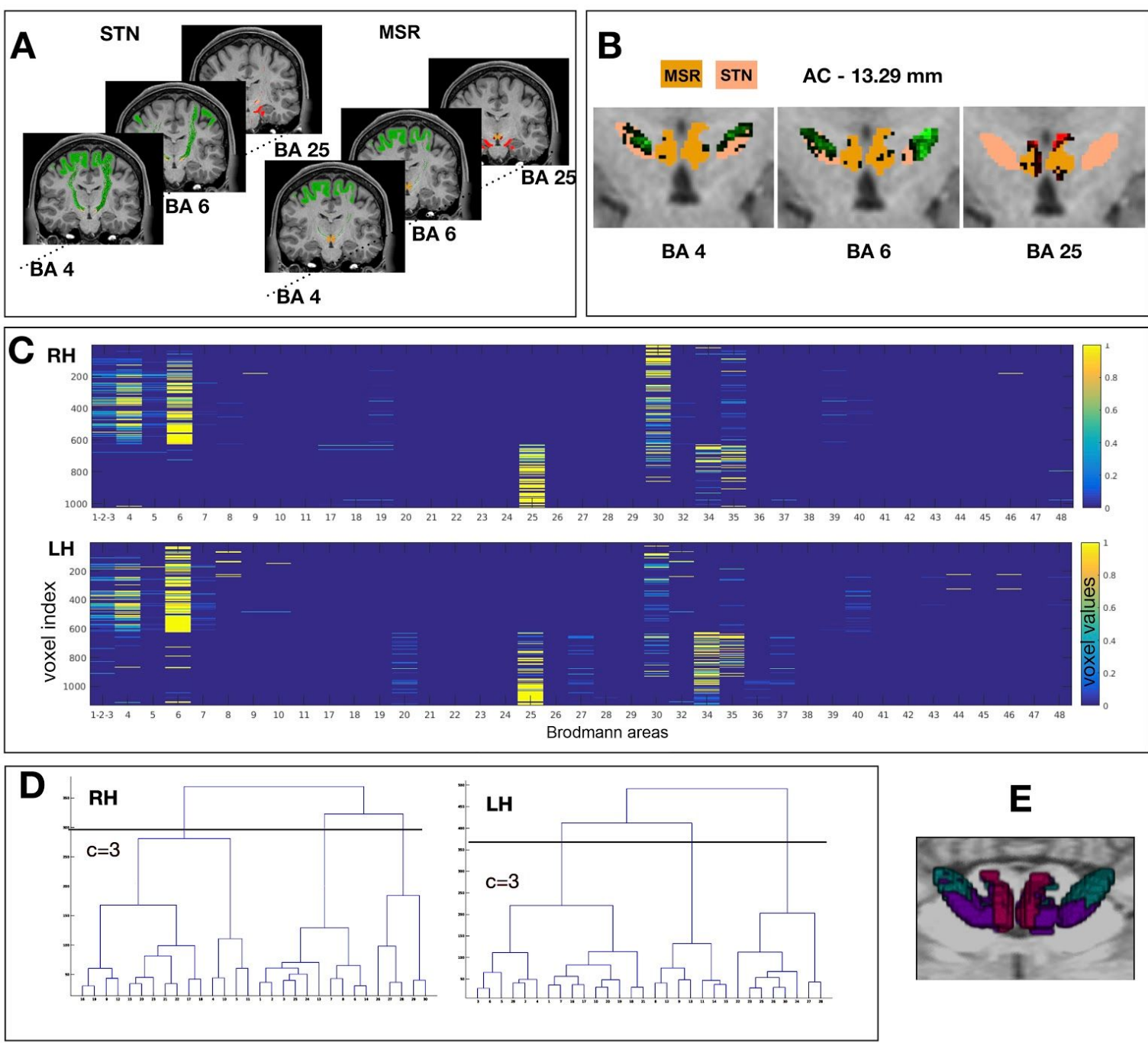

$\mathbf{E}$

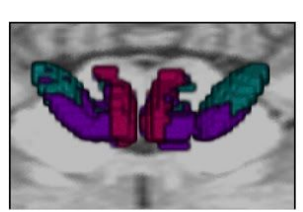

Figure 1: Workflow explaining the steps of clustering the STN and MSR. (A) After whole brain tractography, reconstruction and mask creation, streamlines between 39 cortical masks and both the STN and the MSR were extracted. (B) Corresponding track density maps were 
created. (C) A connectivity matrix was created for each hemisphere as an input to the clustering step. There is one column per cortical area. Each row represents a voxel connectivity profile. Each profile was normalized by its maximum value, therefore color-bar ranges between 0 and 1. (D) The hierarchical tree was cut into 3 clusters. (E) Clustered volumes of the STN and MSR were created within the clustering labels. 